\title{
EFEITO DO MANEJO DA IRRIGAÇÃO E DE POPULAÇÕES DE PLANTAS SOBRE O RENDIMENTO DO ALGODOEIRO HERBÁCEO ${ }^{1}$
}

\author{
FRANCISCO ASSIS DE OLIVEIRA ${ }^{2}$, JOSÉ RENATO CORTEZ BEZERRA ${ }^{3}$ e BERNARDETE CAMPOS OLIVEIRA ${ }^{4}$
}

\begin{abstract}
RESUMO - Objetivou-se estudar, num solo aluvial, franco siltoso, no vale do Açu, no Rio Grande do Norte, o efeito do momento da última irrigação e da população de plantas sobre a altura das plantas e a produtividade do algodoeiro herbáceo (Gossypium hirsutum L.r. latifolium Hutch), cultivar Acala del cerro. Os tratamentos foram definidos pelos momentos da última irrigação aos 65, 80, 95 e 110 dias após a emergência e pela população com 30.000, 60.000, 90.000 e 120.000 plantas/ha. Usou-se o delineamento experimental em blocos ao acaso, com parcelas subdivididas, e quatro repetições. A altura das plantas aumentou com o retardamento da última irrigação e com o tamanho das populações. Houve efeito $(\mathrm{P} \leq 0,01)$ positivo da interação entre os momentos da última irrigação e o tamanho das populações, sobre a produtividade da cultura. O melhor resultado, $4.090 \mathrm{~kg} / \mathrm{ha}$ de algodão em rama, foi proveniente da última irrigação processada aos 95 dias com a população de 90.000 plantas/ha. Últimas irrigações aos 65 e 80 dias foram consideradas como cedo demais, e aos 110 dias, como muito tardias para a produtividade da cultura.
\end{abstract}

Termos para indexação: Gossypium hirsutum, algodão, densidade de plantio, manejo de água.

\section{EFFECT OF IRRIGATION MANAGEMENT AND PLANT POPULATION ON HERBACEOUS COTTON YIELD}

\begin{abstract}
In an alluvial soil, silt loam, of Açu valley, in the state of Rio Grande do Norte, Brazil, a research was carried out to study the effect of time of the last irrigation and plant population on yield and plant height of the herbaceous cotton (Gossypium hirsutum L.r. latifolium Hutch) cultivar Acala del cerro. Treatments consisted of times of the last irrigation at 65, 80,95 and 110 days after emergence and populations with $30,000,60,000,90,000$ and 120,000 plants/ha. The experimental plan was a randomized complete blocks in a split-plot design, with four replications. Delaying time of last irrigation increased height and plant populations. A significant effect $(\mathrm{P} \leq 0.01)$ of interaction between time of last irrigation and plant population was found for cotton yield. The highest cotton yield $(4,090 \mathrm{~kg} / \mathrm{ha})$ was obtained with the interaction between time of last irrigation at 95 days and in population of 90,000 plants/ha. Irrigation times at 65 and 80 days were considered too early, and at 110 days too late for cotton yields.

Index terms: Gossypium hirsutum, cotton plant density, water management.
\end{abstract}

\section{INTRODUÇÃO}

O algodão de sequeiro, representado principalmente pelo algodoeiro arbóreo, durante décadas

\footnotetext{
${ }^{1}$ Aceito para publicação em 13 de maio de 1999.

${ }^{2}$ Eng. Agr., Dr., Prof. Adj., Dep. de Solos e Eng. Rural, Centro de Ciências Agrárias, UFPB, CEP 58397-000 Areia, PB. Bolsista do CNPq. E-mail: oliveira@cca.ufpb.br

${ }^{3}$ Eng. Agr., M.Sc., Embrapa-Centro Nacional de Pesquisa de Algodão (CNPA), Caixa Postal 174, CEP 58107-702 Campina Grande, $P B$.

${ }^{4}$ Bióloga, UEPB, Campus Universitário, CEP 58109-790 Campina Grande, PB. Bolsista PIBIC-CNPq/UEPB
}

figurou como tradicional exploração agrícola pela sua importância socioeconômica em grande parte da região semi-árida do Nordeste brasileiro. Porém, o uso de tecnologias inadequadas, incompatíveis com as irregularidades pluviométricas (Magalhães et al., 1987) e com a propagação do bicudo (Anthonomus grandis Boheman) a partir de 1983, concorreram para praticamente dizimar as culturas do algodoeiro arbóreo na região (Moreira et al., 1989). Isto, de certa forma, tem contribuído para aumentar a área irrigada com o algodoeiro herbáceo, o qual, apesar de ser uma cultura relativamente tole- 
rante ao estresse hídrico, é bem mais sensível do que o algodão arbóreo.

O inter-relacionamento do manejo do solo e da água e a demanda da cultura por uma adequada população de plantas, certamente são fatores determinantes para o sucesso na expansão da área com a cotonicultura irrigada (Al-Khafaf et al., 1978; Fowler, 1979). O manejo inadequado das irrigações é um dos fatores que mais tem limitado o rendimento do algodoeiro, cujo déficit de umidade no solo (Millar, 1976; Guinn et al., 1981) ou o excesso (Levin \& Shmueli, 1964; Bruce \& Romkens, 1965) pode causar redução significativa no rendimento da cultura.

O momento correto para manejar a última irrigação varia com o tipo e as características do solo, com as condições climáticas (Ferry et al., 1976), com o estádio de crescimento e desenvolvimento da cultura, e com a população de plantas ou cobertura vegetal (Bruce \& Romkens, 1965; Al-Khafaf et al., 1978). Estabelecer o momento correto para a irrigação final, no algodoeiro herbáceo, é de grande importância, pois acelera a abertura dos capulhos maduros sem reduzir o rendimento da cultura e a qualidade da fibra (Stockton et al., 1967; Marani, 1973; Ferry et al., 1976). Se o momento da última irrigação for bastante cedo poderá concorrer para reduzir o rendimento da cultura, e para que os capulhos mais novos não atinjam a completa maturação (Bruce \& Shipp, 1962; Levin \& Shmueli, 1964; Miller \& Grimes, 1967; Ferry et al., 1976; Kittock et al., 1983; Oliveira \& Silva, 1987). Porém, se retardar demais a época da última irrigação, poderá favorecer o desenvolvimento vegetativo, atrasar a abertura dos capulhos maduros, retardar a colheita, e aumentar o apodrecimento dos frutos, com reflexos negativos sobre o rendimento e a qualidade do produto (Spooner et al., 1958; Ferry et al., 1976).

Para Fowler (1979), o estabelecimento de um estande adequado do algodoeiro herbáceo é um fator necessário para a cultura atingir seu patamar de rendimento máximo. Porém, para as condições locais do Nordeste, não se dispõe de registros de pesquisas que permitam associar de forma racional o manejo da última irrigação com a população de plantas de algodoeiro herbáceo de forma a maximizar seu rendimento. Nas condições irrigadas do Nordeste brasileiro, a Embrapa (198-) recomenda usar para o algodoeiro herbáceo, cultivar CNPA Acala 1, o espaçamento de $1 \mathrm{~m}$ entre fileiras com $30 \mathrm{~cm}$ entre covas, deixando-se após o desbaste duas plantas/cova (66.000 plantas/ha), ou sete a dez plantas por metro de sulco (70.000 a 100.000 plantas/ha). Portanto, quando se muda apenas o sistema de semeadura, em cova ou em sulco, obtem-se uma diferença de 4.000 a 34.000 plantas/ha. Isto, de certa forma, sinaliza para a escassez de resultados de pesquisa nesta direção, principalmente quando se considera que o algodoeiro herbáceo apresenta hábito de crescimento indeterminado (Beltrão et al., 1994).

Guinn et al. (1981), no Arizona, em condições de déficit de água no solo, constataram que a população de 52.000 plantas/ha de algodão mostrou-se mais produtiva do que a população de 94.000 plantas/ha. Sendouka et al. (1980), com as cultivares de algodoeiro DLT-16, 4S180 e 153F, usando as densidades de $90.000,190.000$ e 360.000 plantas/ha, não encontraram diferença significativa na produção entre cultivares e época de plantio, mas houve efeito de população sobre o rendimento da cultura e a altura de planta. Oliveira \& Silva (1987), usando o sistema de irrigação por sulco na cultura do algodoeiro herbáceo, cultivar SU-0450, para uma população fixa de 57.000 plantas/ha, constataram que a melhor época da última irrigação ocorreu em torno dos 40 dias após o início do florescimento (103 dias do ciclo). Campos et al. (1992), estudando as cultivares de algodoeiro herbáceo CNPA Precoce 1, CNPA Acala 1, CNPA-6H e IAC-20, em um ano de observações, com população fixa de 60.000 plantas/ha, verificaram que houve efeito de cultivar sobre o rendimento, e que aos 95 dias de ciclo seria a melhor época para suspender a última irrigação. Nessas mesmas cultivares, em uma população fixa de 70.000 plantas/ha, Oliveira \& Campos (1997) constataram que a cultura atingiu seu patamar máximo de altura de plantas e de produtividade quando a última irrigação foi realizada aos 80 dias.

O trabalho objetivou avaliar o efeito do momento da última irrigação e da população sobre a altura das plantas e a produtividade da cultura, de algodão herbáceo (Gossypium hirsutum L.r. latifolium Hutch). 


\section{MATERIAL E MÉTODOS}

O trabalho foi desenvolvido durante o período seco do ano (julho/dezembro), na área-piloto da fazenda Umarana, de propriedade do Grupo Finobrasa, no vale do Açu, a $10 \mathrm{~km}$ ao norte da cidade de Açu, RN. O local está delimitado pelas coordenadas geográficas: entre as latitudes $5^{\circ}$ e $6^{\circ} \mathrm{S}$ e entre as longitudes $36^{\circ}$ e $37^{\circ} \mathrm{W}$.G. e altitude de 68 m. Segundo a classificação de Köppen (Sudene, 1971), o clima da região é do tipo Bsw'h' (clima muito quente e semi-árido, tipo estepe). Porém, para Hagreaves (1974), o clima local é do tipo seco úmido, com precipitação média anual de $870 \mathrm{~mm}$, apresentando duas estações bem definidas, uma, chuvosa, de janeiro a junho, e outra, seca, de julho a dezembro, com temperatura média anual de $24,8^{\circ} \mathrm{C}$

Segundo as frações granulométricas, o solo da área foi classificado como aluvial, franco siltoso, apresentando nos $30 \mathrm{~cm}$ superficiais do perfil $400 \mathrm{~g} / \mathrm{dm}^{3}$ de areia total, $550 \mathrm{~g} / \mathrm{dm}^{3}$ de silte, $50 \mathrm{~g} / \mathrm{dm}^{3}$ de argila, $\mathrm{pH} \mathrm{7,1} \mathrm{e}$ $82 \mathrm{mg} / \mathrm{dm}^{3} \mathrm{de} \mathrm{P}, 103,59,4$ e $10 \mathrm{mmol}_{\mathrm{d}} / \mathrm{dm}^{3} \mathrm{de} \mathrm{Ca}^{2+}, \mathrm{Mg}^{2+}$, $\mathrm{K}^{+} \mathrm{e} \mathrm{Na}^{+}$respectivamente, e $12 \mathrm{~g} / \mathrm{dm}^{3}$ de matéria orgânica A área recebeu uma adubação de manutenção nos sulcos de semeadura constituída de $40 \mathrm{~kg} / \mathrm{ha}$ de N-sulfato de amônio, $50 \mathrm{~kg} / \mathrm{ha}$ de $\mathrm{P}_{2} \mathrm{O}_{5}$-superfosfato simples, e $30 \mathrm{~kg} / \mathrm{ha}$ de $\mathrm{K}_{2} \mathrm{O}$-cloreto de potássio, e em cobertura foram aplicadas duas doses de $30 \mathrm{~kg} / \mathrm{ha}$ de $\mathrm{N}$, aos 25 e 45 dias após a emergência. O preparo da área constou de aração e duas gradagens niveladoras em sentidos cruzados

Os tratamentos foram definidos pelos momentos de última irrigação aos $65,80,95$ e 110 dias após a emergência e pelas populações de 30, 60, 90 e $120 \mathrm{mil}$ plantas/ha, designadas por baixa (B), normal (N), alta (A) e muito alta (MA), respectivamente. Como cultura-teste foi usado o algodoeiro herbáceo (Gossypium hirsutum L.r. latifolium Hutch), cultivar Acala del cerro. Utilizou-se espaçamento de $1 \mathrm{~m}$ entre fileiras contínuas, colocando-se para germinar, em média, 25 sementes por metro de sulco e 15 dias após a emergência foi processado o desbaste, deixando-se em cada tratamento a população de plantas preestabelecida. As sementes foram semeadas em 21 de agosto de 1992, as plântulas emergiram 9 dias depois, e a colheita final foi realizada em 12 de janeiro de 1993 . O delineamento experimental utilizado foi em blocos ao acaso, com parcelas subdivididas, e quatro repetições. As parcelas foram constituídas pelo momento da última irrigação, e as subparcelas, pelas populações de plantas. A área total da parcela principal foi de $96 \mathrm{~m}^{2}$ ( $16 \mathrm{~m}$ de comprimento x $6 \mathrm{~m}$ de largura) e a subparcela $24 \mathrm{~m}^{2}$ (4 m de comprimento x $6 \mathrm{~m}$ de lagura), ficando como área útil os $10 \mathrm{~m}^{2}$ da parte central da subparcela. A cultura foi mantida livre de plantas daninhas por meio de capinas manuais, e de pragas, por meio de pulverizações preventivas.

Usou-se o sistema de irrigação por aspersão convencional, de média pressão, com tubulações de alumínio, sendo a rede principal de $100 \mathrm{~mm}$ de diâmetro, e a secundária, de $62 \mathrm{~mm}$. Os aspersores eram de marca Fibramar A252 com diâmetro do bocal 5,6 mm, pressão de serviço de $2,0 \mathrm{~kg} / \mathrm{cm}^{2}$, espaçamento de $12 \mathrm{~m} \mathrm{x} 12 \mathrm{~m} \mathrm{e}$ precipitação de $11,5 \mathrm{~mm} /$ hora. Fez-se o controle das irrigações, com base na água disponível no solo e na evapotranspiração da cultura, determinada indiretamente em função da evaporação do tanque classe A e do coeficiente da cultura recomendado por Hargreaves (1956). Com relação à umidade disponível no solo, considerou-se a água no solo retida entre as tensões de 33 e $1.500 \mathrm{kPa}$ (Fig. 1). O manejo da irrigação foi processado sempre que a evapotranspiração da cultura atingia, em média, $50 \%$ da umidade disponível nos $60 \mathrm{~cm}$ superficiais do solo.

Para interpretar o efeito dos tratamentos, foram computados os dados de altura das plantas por ocasião da colheita, e de produtividade de algodão em rama; os resultados foram submetidos a análise de variância, e as médias foram comparadas entre si, pelo teste de Tukey a $5 \%$ de probabilidade.

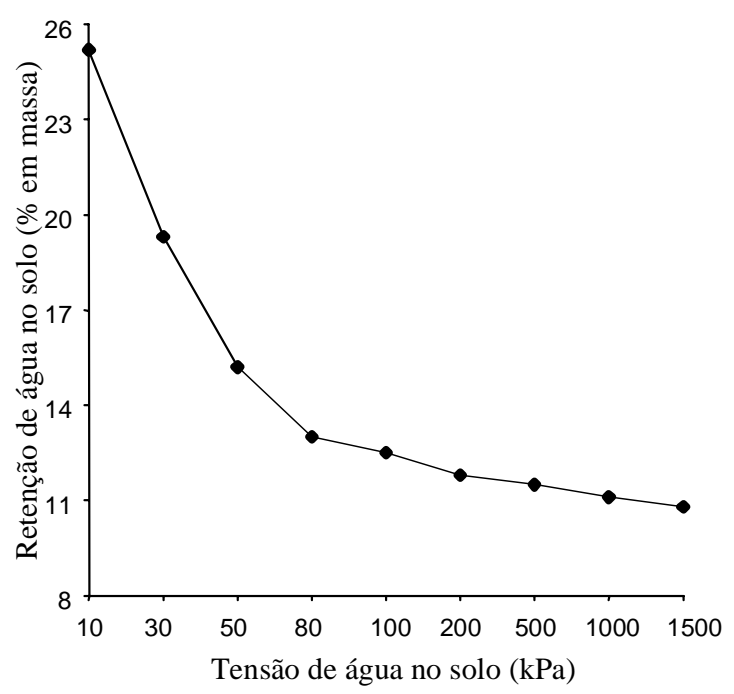

FIG. 1. Curva característica de retenção de água no solo da área onde foi conduzido o ensaio. 


\section{RESULTADOS E DISCUSSÃO}

Os resultados da análise de variância encontramse na Tabela 1, onde se observa que houve efeito altamente significativo $(p \leq 0,01)$ dos tratamentos sobre a altura das plantas e a produtividade do algodão em rama. Além dos efeitos isolados tanto dos momentos da última irrigação quanto das populações de plantas, houve também efeito da interação desses fatores sobre os resultados das variáveis analisadas.

A Tabela 2 resume os resultados de altura de plantas, onde é possível observar que houve, em geral, uma tendência em cada nível de população, de as plantas crescerem à medida que se retardou o momento da última irrigação. Nas populações baixa e normal, as maiores alturas de plantas, $108,7 \mathrm{~cm}$ e
$111,1 \mathrm{~cm}$, respectivamente, foram atingidas quando a última irrigação foi aplicada aos 110 dias. Na população alta, as maiores alturas das plantas foram atingidas com a última irrigação conduzida aos $110 \mathrm{e}$ 95 dias, indiferentemente. Comportamento semelhante ocorreu com a população muito alta, exceto nas condições em que a última irrigação foi aplicada aos 65 e 80 dias que apresentaram resultados de altura de plantas significativamente diferentes entre si. Considerando os resultados das alturas das plantas referentes às populações dentro de cada momento da última irrigação, não houve efeito significativo das populações sobre a altura das plantas em relação à última irrigação aos 65 ou 110 dias. Porém, no tratamento 80 dias, a maior altura da planta foi $93,3 \mathrm{~cm}$, proveniente da população muito alta e na irrigação aos 95 dias, as alturas das plantas das populações

TABELA 1. Análise de variância (quadrado médio) referente aos resultados de produtividade de algodão em rama e de altura de planta, em algodoeiro herbáceo, cultivar Acala del cerro.

\begin{tabular}{|c|c|c|c|}
\hline \multirow[t]{2}{*}{ Causa da variação } & \multirow[t]{2}{*}{ GL } & \multicolumn{2}{|c|}{ Quadrado médio } \\
\hline & & Produtividade & Altura \\
\hline Blocos & 3 & $51.821,6^{\mathrm{ns}}$ & $48,8^{\mathrm{ns}}$ \\
\hline Momento (M) & 3 & $9.287 .875,4^{* *}$ & $3.155,1^{* *}$ \\
\hline Resíduo (a) & 9 & $21.215,5$ & 13,1 \\
\hline Parcela & (15) & $(1.880 .668,6)$ & $(648,6)$ \\
\hline População (P) & 3 & $3.307 .934,1^{* *}$ & $53,2^{* *}$ \\
\hline Interação MxP & 9 & $312.346,3^{* *}$ & $34,6^{* *}$ \\
\hline Resíduo (b) & 36 & $17.335,2$ & 11,0 \\
\hline \multicolumn{4}{|l|}{ Desdobram. de $\mathrm{P}$ dentro de $\mathrm{M}$} \\
\hline P. dentro de $\mathrm{M}(1)$ & 3 & $294.336,5^{* *}$ & $4,7^{\mathrm{ns}}$ \\
\hline P. dentro de $\mathrm{M}(2)$ & 3 & $531.235,7 * *$ & $72,7 * *$ \\
\hline P. dentro de $\mathrm{M}(3)$ & 3 & $2.319 .058,2 * *$ & $65,7 * *$ \\
\hline P. dentro de $\mathrm{M}(4)$ & 3 & $1.100 .342,7 * *$ & $13,8^{\mathrm{ns}}$ \\
\hline Resíduo (c) & 36 & $17.335,2$ & 11,0 \\
\hline \multicolumn{4}{|l|}{ Desdobram. de $\mathrm{M}$ dentro de $\mathrm{P}$} \\
\hline M. dentro de $\mathrm{P}(1)$ & 3 & $691.599,7 * *$ & $611,5 * *$ \\
\hline M. dentro de $\mathrm{P}(2)$ & 3 & $3.310 .778,1 * *$ & $727,4 * *$ \\
\hline M. dentro de $\mathrm{P}(3)$ & 3 & $4.018 .009,5^{* *}$ & $1.023,5 * *$ \\
\hline M. dentro de $\mathrm{P}(4)$ & 3 & $2.204 .527,2^{* *}$ & $896,4 * *$ \\
\hline Resíduo (d) & 42 & $18.305,3$ & 11,5 \\
\hline C.V. (\%) para momento (M) & & 5,37 & 3,74 \\
\hline C.V. (\%) para população $(\mathrm{P})$ & & 4,86 & 3,44 \\
\hline $\mathrm{dms}^{1}$ para momento & & 160,9 & 4,0 \\
\hline dms para população & & 125,4 & 3,2 \\
\hline
\end{tabular}

${ }^{1}$ Diferença mínima significativa pelo teste de Tukey a $5 \%$ de probabilidade

ns e ** Não-significativo e significativo a $1 \%$ de probabilidade, respectivamente, pelo teste $\mathrm{F}$ 
alta e muito alta foram superiores às das populações baixa e normal.

Dos resultados obtidos, é possível afirmar que o aumento na população de plantas sinaliza para o retardamento da última irrigação até aos 95 dias, a fim de a cultura completar seu crescimento, e que, prolongando a última irrigação para os 110 dias, o efeito decorrente de população de plantas deixa de existir. Independentemente do tamanho da população de plantas, o momento da última irrigação aos 65 ou 80 dias foi cedo demais para a cultura completar seu crescimento normal. Oliveira \& Campos (1997), trabalhando em condições similares, com outras cultivares de algodoeiro herbáceo, sob irrigação por sulcos, concordam que a última irrigação aos 65 dias seja cedo demais para a cultura atingir seu completo crescimento, mas observam que aos 80 dias não é cedo o suficiente para limitar a altura das plantas. Porém, Sendouka et al. (1980), na Grécia, em condições não irrigadas, com três cultivares de algodoeiro herbáceo constataram que o aumento na densidade de plantas contribuiu para reduzir significativamente o crescimento da cultura.

Nas populações baixa e normal, os maiores rendimentos da cultura, foram obtidos aplicando-se a última irrigação aos 95 e 110 dias. Nas populações alta e muito alta, os melhores resultados foram conseguidos com a última irrigação aos 95 dias (Tabela 3). Pode-se afirmar que em todos os níveis de população de plantas os momentos da última irrigação aos 65 e 80 dias foram cedo demais para a cultura atingir seu patamar de produtividade, e que a última irrigação aos 110 dias foi considerada tardia, e portanto concorreu para limitar a produção da cultura. Porém, os trabalhos de Oliveira \& Campos (1997), em condições similares, com as cultivares de algodoeiro herbáceo CNPA Precoce 1, CNPA Acala 1, CNPA-6H e IAC-20, concordam que aos 95 dias seja o momento apropriado para a última irrigação, mas discordam que aos 80 dias seja cedo demais e 110 dias muito tardio para o momento da última irrigação. Por outro lado, Kittock et al. (1983), no Arizona, constataram que a última irrigação aos 108 dias foi cedo demais para a cultura do algodoeiro atingir produtividade significativamente elevada.

Dentro de cada momento de última irrigação (Tabela 3), pode-se constatar que, aos 65 dias, o maior rendimento da cultura foi proveniente da população muito alta; aos 80 dias os melhores resultados foram os das populações muito alta e alta; aos 95 dias o maior rendimento foi o da população alta e aos 110 dias as populações muito alta, alta e normal apresentaram rendimentos superiores ao da população baixa. O efeito de populações de plantas, embora em condições de precipitação natural, também foi obtido por Sendouka et al. (1980), na Grécia, quando, testando as densidades de 90.000, 190.000 e 360.000 plantas/ha, constataram que 190.000 plantas/ha apresentou o melhor resultado de produtividade de algodão em rama.

É possível afirmar que, nos momentos de última irrigação, inferiores a 95 dias, independentemente das populações de plantas utilizadas, os resultados de produtividade da cultura foram significativamente reduzidos. Vários pesquisadores (Spooner, 1958; Stockton et al., 1967; Ferry et al., 1976; Kittock

TABELA 2. Altura da planta em diversas populações de plantas em razão do momento da última irrigação (dias após emergência), em algodão herbáceo, cultivar Acala del cerro'

\begin{tabular}{|c|c|c|c|c|c|}
\hline \multirow{2}{*}{$\begin{array}{l}\text { Última irrigação } \\
\text { (dias) }\end{array}$} & \multicolumn{5}{|c|}{ População $^{2}$ (mil plantas/ha) } \\
\hline & $30(\mathrm{~B})$ & $60(\mathrm{~N})$ & $90(\mathrm{~A})$ & 120 (MA) & $\mathrm{dms}$ \\
\hline 65 & $83,0 \mathrm{cA}$ & $82,7 \mathrm{cA}$ & $83,0 \mathrm{bA}$ & $80,7 \mathrm{cA}$ & 6,3 \\
\hline 80 & $86,3 \mathrm{cB}$ & $86,0 \mathrm{cB}$ & $83,3 \mathrm{bB}$ & $93,3 \mathrm{bA}$ & 6,3 \\
\hline 95 & $102,0 \mathrm{bB}$ & $102,7 \mathrm{bB}$ & $109,3 \mathrm{aA}$ & $109,3 \mathrm{aA}$ & 6,3 \\
\hline 110 & $108,7 \mathrm{aA}$ & $111,1 \mathrm{aA}$ & $112,3 \mathrm{aA}$ & $113,0 \mathrm{aA}$ & 6,3 \\
\hline $\mathrm{dms}$ & 6,4 & 6,4 & 6,4 & 6,4 & \\
\hline
\end{tabular}


TABELA 3. Valores médios de produtividade de algodão em rama em razão de diferentes momentos da última irrigação (dias após emergência) e populações de plantas do algodoeiro herbáceo, cultivar Acala del cerro ${ }^{1}$.

\begin{tabular}{|c|c|c|c|c|c|}
\hline \multirow{2}{*}{$\begin{array}{l}\text { Última irrigação } \\
\text { (dias) }\end{array}$} & \multicolumn{4}{|c|}{ População ${ }^{2}$ (mil plantas/ha) } & \multirow[t]{2}{*}{ dms } \\
\hline & $30(\mathrm{~B})$ & $60(\mathrm{~N})$ & $90(\mathrm{~A})$ & 120 (MA) & \\
\hline & & 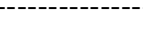 & - (kg/ha) - & & 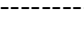 \\
\hline 65 & $1.595 \mathrm{bC}$ & $1.637 \mathrm{cBC}$ & $1.866 \mathrm{~dB}$ & $2.187 \mathrm{dA}$ & 250,8 \\
\hline 80 & $1.850 \mathrm{bC}$ & $2.272 \mathrm{bB}$ & $2.580 \mathrm{cA}$ & $2.651 \mathrm{cA}$ & 250,8 \\
\hline 95 & $2.351 \mathrm{aD}$ & $3.462 \mathrm{aC}$ & $4.090 \mathrm{aA}$ & $3.801 \mathrm{aB}$ & 250,8 \\
\hline 110 & $2.477 \mathrm{aB}$ & $3.468 \mathrm{aA}$ & $3.607 \mathrm{bA}$ & $3.480 \mathrm{bA}$ & 250,8 \\
\hline dms & 255,9 & 255,9 & 255,9 & 255,9 & \\
\hline
\end{tabular}

${ }^{1}$ Médias seguidas da mesma letra, maiúsculas na linha e minúsculas na coluna, não diferem significativamente entre si, pelo teste de Tukey a $5 \%$ de probabilidade; dms: diferença mínima significativa

2 Letras entre parênteses se referem às populações baixa $(B)$, normal $(N)$, alta $(A)$ e muito alta $(M A)$

et al., 1983) concordam que o momento inadequado para a última irrigação concorre para reduzir significativamente a produtividade da cultura do algodão.

\section{CONCLUSÕES}

1. O crescimento da cultura é favorecido pelo retardamento da época da última irrigação e pelo aumento da população de plantas.

2. A última irrigação aos 80 dias é cedo demais, e aos 110 dias é tarde demais para o algodão atingir seu patamar de rendimento máximo.

3. A combinação da última irrigação aos 95 dias após a emergência e a população de 90.000 plantas/ha promove o melhor rendimento de algodão em rama.

\section{REFERÊNCIAS}

AL-KHAFAF, S.; WIERENGA, P.J.; WILLIAMS, B.C. Evaporative flux from irrigated cotton as related to leaf area index, soil water, and evaporative demand Agronomy Journal, Madison, v.70, n.6, p. 912-917, 1978 .

BELTRÃO, N.E. de M.; SOUZA, J.G. de; AZEVÊDO, D.M.P. de; NÓBREGA, L.B. da. Plasticidade morfológica do algodoeiro herbáceo em função da queda induzida das estruturas de reprodução. Campina Grande: Embrapa-CNPA, 1994. 40p. (Embrapa-CNPA. Documentos, 40).
BRUCE, R.R.; ROMKENS, M.J.M. Fruiting and growth characteristics of cotton in relation to soil moisture tension. Agronomy Journal, Madison, v.57, n.2, p.135-140, 1965.

BRUCE, R.R.; SHIPP, C.D. Cotton fruiting affected by soil moisture regime. Agronomy Journal, Madison, v.54, n.1, p.15-18, 1962.

CAMPOS, T.G. da; OLIVEIRA, F.A. de; SANTOS, J.W dos. Estudo da última irrigação em quatro cultivares de algodoeiro herbaceo. In: CONGRESSO NACIONAL DE IRRIGAÇÃO E DRENAGEM, 9., 1991, Natal. Anais. Fortaleza: Associação Brasileira de Irrigação e Drenagem, 1992. p.1689-1702.

EMBRAPA. Centro Nacional de Pesquisa do Algodão (Campina Grande, PB). "CNPA Acala 1". Nova cultivar de algodoeiro herbáceo de fibra longa para áreas irrigadas do Nordeste. Campina Grande, [198-]. Folder

FERRY, G.V.; GEORGE, A.G.; JOHNSON, C.E.; McCUTCHEON, O.D.; STROMBERG, L.K.; BOOHER, L.J.; HOOVER, M. Guides in cotton irrigation. California: Univ. of California, 1976. 25p

FOWLER, J.L. In-furrow water injection for improving cotton stand establishment. Agronomy Journal, Madison, v.71, n.3, p.453-457, 1979.

GINN, G.; MAUNEY, J.R.; FRY, K.E. Irrigation scheduling and plant population effects on growth, 
bloom rates, boll abscission, and yield of cotton Agronomy Journal, Madison, v.73, n.3, p.529-534, 1981.

HARGREAVES, G.H. Irrigation requirement based on climatic data. Journal of Irrigation and Drainage Division, Washington, v.1105, p.1-10, 1956.

HARGREAVES, G.H. Potential evapotranspiration and irrigation requirements for Northeast Brazil. Utah: Utah Univ., 1974. 55p.

KITTOCK, D.L.; HENNEBERRY, T.J.; BARIOLA, L.A.; TAYLOR, B.B.; HOFMAN, W.C. Cotton boll period response to water stress and pink bollworm Agronomy Journal, Madison, v.75, n.1, p.17-20, 1983

LEVIN, I.; SHMUELI, E. The response of cotton to various irrigation regimes in the Hula Valley. Israel Journal of Agriculture Research, Bet Dagan, v.14, p.211-225, 1964.

MAGALHÃES, A.R.; CHAIB FILHO, H.; GARAGORRY, F.L.; GASQUE, J.G.; MOLION, L.C.B.; AMORIM NETO, M. da S; NOBRE, C.A; PORTO, E.R.; REBOUÇAS, O.E. The effects of climatic variations on agriculture in Northeast of Brazil. Luxemburg : International Institute for Applied Systems Analysis, 1987. 109p.

MARANI, A. Effects of soil moisture stress on two varieties of upland cotton in Israel. IV. Effects of periods of stress occurrence, correlations and regressions. Experimental Agriculture, London, v.9 n. 2, p. $121-128,1973$

MILLAR, A.A. Respuesta de los cultivos al déficit de agua como información básica para el manejo del riego. Petrolina: Embrapa-CPATSA, 1976. 62p.

MILLER, R.J.; GRIMES, D.W. Effects of moisture stress on cotton yields. California Agriculture, Berkeley, v. 2, n. 8, p. $18-19,1967$
MOREIRA, J.A.N.; FREIRE, D.E.; SANTOS, R.F. dos; BARREIRO NETO, M. Algodoeiro mocó: uma lavoura ameaçada de extinção. Campina Grande: Embrapa-CNPA, 1989. 20p. (Embrapa-CNPA. Documentos, 36)

OLIVEIRA, F.A. de; CAMPOS, T.G. da S. Manejo da irrigação na cultura do algodoeiro herbáceo em condições semi-áridas do Nordeste. Pesquisa Agropecuária Brasileira, Brasília, v.32, n.5, p.521-531, maio 1997.

OLIVEIRA, F.A. de; SILVA, J.J.S. Efeito da última irrigação e número de colheita na cultura do algodão. Salvador : EPABA/UEP S.F., 1987. 27p. (EPABA. Boletim de Pesquisa, 7).

SENDOUKA, S.G.; SFICAS, A.G.; FOTIADIS, N.A.; GAGIANAS, A.A.; GERAKIS, P.A. Effect of population density, planting date, and genotype on plant growth and development of cotton. Agronomy Journal, Madison, v.72, n.2, p.347-353, 1980 .

SPOONER, A.E.; CAVINESS, C.E.; SPURGEON, W.I Influence of timing of irrigation on yield, quality and fruiting of Upland cotton. Agronomy Journal, Madison, v.50, n.1, p.74-77, 1958.

STOCKTON, J.R.; CARREKER, J.R.; HOOVER, M. Sugar, oil and fiber crops. Parte IV - Irrigation of cotton and other fiber. In: HAGAN, R.M.; EMISTER, T.W. (Eds.). Irrigations of agricultural lands. Madison: American Society of Agronomy, 1967. p.161-171 (Agronomy, 11)

SUDENE. Levantamento exploratório - reconhecimento dos solos do Estado do Rio Grande do Norte. Rio de Janeiro, 1971. 531p. (Boletim Técnico, 21, Série pedológica, 9). 